\title{
Bone Formation in a Rat Tibial Defect Model Using Carboxymethyl Cellulose/BioC/Bone Morphogenic Protein-2 Hybrid Materials
}

\author{
Sang-Heon Song, ${ }^{1}$ Young-Pil Yun, ${ }^{2}$ Hak-Jun Kim, ${ }^{2}$ \\ Kyeongsoon Park, ${ }^{3}$ Sung Eun Kim, ${ }^{2}$ and Hae-Ryong Song ${ }^{2}$ \\ ${ }^{1}$ Department of Orthopedic Surgery, Myongji Hospital, 55 Hwasu-ro 14 Beon-gil, Deokyang-gu, \\ Goyang 412-826, Republic of Korea \\ ${ }^{2}$ Department of Orthopedic Surgery and Rare Diseases Institute, Korea University Medical College, Guro Hospital, \\ No. 80, Guro-dong, Guro-gu, Seoul 152-703, Republic of Korea \\ ${ }^{3}$ Division of Bioimaging, Chuncheon Center, Korea Basic Science Institute, 192-1 Hyoja 2-Dong, Chuncheon, \\ Gangwon-do 200-701, Republic of Korea
}

Correspondence should be addressed to Sung Eun Kim; sekim10@korea.ac.kr and Hae-Ryong Song; songhae@korea.ac.kr Received 9 November 2013; Accepted 12 March 2014; Published 6 April 2014

Academic Editor: Guoyan Zheng

Copyright (C) 2014 Sang-Heon Song et al. This is an open access article distributed under the Creative Commons Attribution License, which permits unrestricted use, distribution, and reproduction in any medium, provided the original work is properly cited.

\begin{abstract}
The objective of this study was to assess whether carboxymethyl cellulose- (CMC-) based hydrogel containing BioC (biphasic calcium phosphate (BCP); tricalcium phosphate (TCP) : hydroxyapatite (Hap) $=70: 30)$ and bone morphogenic protein-2 (BMP2) led to greater bone formation than CMC-based hydrogel containing BioC without BMP-2. In order to demonstrate bone formation at 4 and 8 weeks, plain radiographs, microcomputed tomography (micro-CT) evaluation, and histological studies were performed after implantation of all hybrid materials on an $8 \mathrm{~mm}$ defect of the right tibia in rats. The plain radiographs and micro$\mathrm{CT}$ analyses revealed that $\mathrm{CMC} / \mathrm{BioC} / \mathrm{BMP}-2(0.5 \mathrm{mg})$ led to much greater mineralization at 4 and 8 weeks than did $\mathrm{CMC} / \mathrm{BioC}$ or CMC/Bio/BMP-2 (0.1 mg). Likewise, bone formation and bone remodeling studies revealed that CMC/BioC/BMP-2 (0.5 mg) led to a significantly greater amount of bone formation and bone remodeling at 4 and 8 weeks than did $\mathrm{CMC} / \mathrm{BioC}$ or $\mathrm{CMC} / \mathrm{BioC} / \mathrm{BMP}-2$ $(0.1 \mathrm{mg})$. Histological studies revealed that mineralized bone tissue was present around the whole circumference of the defect site with CMC/BioC/BMP-2 $(0.5 \mathrm{mg})$ but not with CMC/BioC or CMC/BioC/BMP-2 $(0.1 \mathrm{mg})$ at 4 and 8 weeks. These results suggest that $\mathrm{CMC} / \mathrm{BioC} / \mathrm{BMP}-2$ hybrid materials induced greater bone formation than CMC/BioC hybrid materials. Thus, CMC/BioC/BMP-2 hybrid materials may be used as an injectable substrate to regenerate bone defects.
\end{abstract}

\section{Introduction}

More than a million people in the United States and other countries require bone graft to regenerate bone defects caused by fracture, trauma, or tumor resection $[1,2]$. Autograft has been used widely as bone graft due to its excellent osteoinductive and osteoconductive properties [2]. Allograft has also been used as a bone substitute because it prevents donor site morbidity [2]. However, both autograft and allograft have disadvantages, such as limited availability, donor site morbidity, transmission of infectious diseases, and immunerejection reactions $[2,3]$. To address these shortcomings, a new approach to bone graft has been developed.

Hydroxyapatite (HAp) and calcium phosphate (CaP) have been successfully used as bone graft materials for the regeneration of bone defects due to their physical and chemical properties and their structural similarity to natural bone. CaP-based materials have been approved by the Food and Drug Administration (FDA) for clinical applications in the fields of dental and orthopedic surgery. CaP-based materials 
possess bioactive, biocompatible, and osteoconductive properties [4]. Although CaP-based materials have been widely used in a clinical setting, they have no osteogenic or osteoinductive properties; however, such properties are needed for the repair and regeneration of bone defects. The osteoinductive properties of natural bone are the result of bone morphogenic proteins (BMPs) and osteogenic proteins that exist in the extracellular matrix (ECM) [5-9]. Thus, in order to produce more effective bone regeneration, CaP-based materials combined with osteoinductive materials are required.

BMPs have osteoinductive properties. Among BMP family members, BMP-2 is one of the most osteoinductive growth factors. Previous studies have demonstrated that BMP-2 can induce mesenchymal stem cells (MSCs) to differentiate into osteoblast lineages and regenerate bone [1013]. In addition, previous studies have shown that BMP-2 stimulates osteogenic markers such as osteopontin, osteocalcin, bone sialoprotein, and alkaline phosphatase (ALP) during osteogenic differentiation in vitro $[14,15]$. Recently, to enhance osteoblast function and bone formation, BMP-2based delivery systems have been developed. Previous studies have reported that BMP-2 combined with collagen gels, sponges, scaffolds, hyaluronic acid, dextran, chitosan, and fibrin scaffolds induces the regeneration of bone defects [16-21].

Carboxymethyl cellulose (CMC) has natural biodegradable and biocompatible properties $[22,23]$ and has been used as a biomedical membrane $[24,25]$. In an in vitro study, Leone et al. demonstrated that amidated carboxymethyl cellulose (CMCA) hydrogel is a potential filler for cartilage defects [26]. Moreover, a recent report demonstrated that a CMC/HAp hybrid hydrogel induced more osteoblast-like cell proliferation, osteogenic markers including Runx2, ALP, and collagen type I, and mineralization than did a CMC-based hydrogel without HAp [27].

On the basis of these results, we hypothesized that a CMC-based hydrogel containing BioC (biphasic calcium phosphate (BCP); tricalcium phosphate (TCP): hydroxyapatite (Hap) $=70: 30)$ and BMP-2 would promote greater bone formation in a rat tibial defect model than would a CMCbased hydrogel containing BioC without BMP-2.

\section{Materials and Methods}

2.1. Release Kinetics of BMP-2 from $C M C / B i o C / B M P-2$ Hybrid Materials. The cellulose material (CMC-based hydrogel containing BioC) has been integrated with two different BMP2 solutions $(0.1 \mathrm{mg}$ and $0.5 \mathrm{mg}$; Cowellmedi Co., Busan, Korea) with a simple soaking manner. Released BMP-2 from CMC/BioC/BMP-2 (0.1 mg) and CMC/Bio-C/BMP-2 $(0.5 \mathrm{mg})$ hybrid materials was evaluated with an enzymelinked immunosorbent assay (ELISA) in accordance with the manufacturer's instructions by using a microplate reader (Bio-Rad, Hercules, CA, USA) at a wavelength of $450 \mathrm{~nm}$. In brief, CMC/BioC/BMP-2 (0.1 mg) and CMC/BioC/BMP$2(0.5 \mathrm{mg})$ blended materials were, respectively, soaked in a membrane bag (MWCO: 300,000), $15 \mathrm{~mL}$ tube with $1 \mathrm{~mL}$ of phosphate buffer saline (PBS) (Gibco BRL, Rockville, MD, USA). The tube was incubated at $37^{\circ} \mathrm{C}$ with gentle shaking at $100 \mathrm{rpm}$. At predetermined time intervals of $1,3,5$, and $10 \mathrm{hr}$ and $1,3,5,7,14,21$, and 28 days, supernatants were collected and replaced with fresh PBS for 28 days. The absorbance of the collected samples was determined with a microplate reader.

\subsection{Animal Study}

2.2.1. Rat Treatments. Eight-week-old Sprague-Dawley rats (Orient Bio Co., Seongnam City, Korea) were used for the in vivo evaluation of $\mathrm{CMC} / \mathrm{BioC}, \mathrm{CMC} / \mathrm{BioC} / \mathrm{BMP}-2$ (0.1 mg), and CMC/BioC/BMP-2 (0.5 mg) groups. The experimental protocol was approved by the Institutional Animal Care and Use Committee of the Korea University Medical Center (KUIACUC-2012-128). Experimental animals were divided into three groups: group I $(n=4)$ was implanted with $\mathrm{CMC} / \mathrm{BioC}$, group II $(n=4)$ was implanted with CMC/BioC/BMP-2 (0.1 mg), and group III $(n=4)$ was implanted with CMC/BioC/BMP-2 $(0.5 \mathrm{mg})$. The rats were anesthetized with tiletamine/zolazepam (50 mg/kg; Zoletil) and xylazine (10 mg/kg; Rompun). After shaving the right tibia, the periosteum and soft tissue were carefully retracted and two $0.9 \mathrm{~mm} \mathrm{~K}$-wires (Zimmer, Warsaw, IN) were fixed to the right tibia. K-wires were clamped bilaterally with the author's own-designed external fixator (U\&I, Gyeonggi-do, Korea). An $8 \mathrm{~mm}$ defect of the right tibia was created with a cutting burr, and $150 \mu \mathrm{L}$ of $\mathrm{CMC} / \mathrm{BioC}, \mathrm{CMC} / \mathrm{BioC} / \mathrm{BMP}-$ $2(0.1 \mathrm{mg})$, or CMC/BioC/BMP-2 $(0.5 \mathrm{mg})$ was injected into the right tibia defect, as appropriate, respectively. The subcutaneous tissue and skin were sutured with absorbable 40 vicryl (Ethicon, Somerville, NJ, USA) (Figure 1). The rats were allowed free movement in cages after recovery from anesthesia. One rat in group I had been dead for two days after treatment, so another rat has been treated with the same manners with others in group I.

\subsubsection{Bone Formation Analyses}

Plain Radiographs. At 4 and 8 weeks after injection, samples were fixed in $3.7 \%$ paraformaldehyde solution. Radiographs of the specimens were obtained with a plain radiograph apparatus (In Vivo DXS 4000 Pro System, Carestream Health, Rochester, NY, USA) at $43 \mathrm{KVP}, 2 \mathrm{~mA}$, and $44 \mathrm{~cm}$ filmradiation beam distance for a $1.5 \mathrm{~s}$ exposure time.

Microcomputed Tomography (Micro-CT) Evaluation. At predetermined time intervals of 4 and 8 weeks, bone volume was obtained with a micro-CT system (Albira II Imaging System, Carestream Health). The CT system was operated at a voltage of $40 \mathrm{kV}$, and a current of $250 \mu \mathrm{A}$ was used with a nominal resolution of $9 \mu \mathrm{m} /$ pixel. Image analysis was performed with a bone analyzer (Molecular Imaging Analysis software; Carestream Health Inc., Woodbridge, VA, USA).

Histological Study. The specimens were retrieved at 4 and 8 weeks. After decalcification, the samples were embedded in paraffin. The tissues were cross-sectioned at an $8 \mu \mathrm{m}$ thickness in the longitudinal parallel direction and stained with hematoxylin and eosin (H\&E) and Masson's trichrome staining. The cytoplasm of osteoblasts and bone formation 


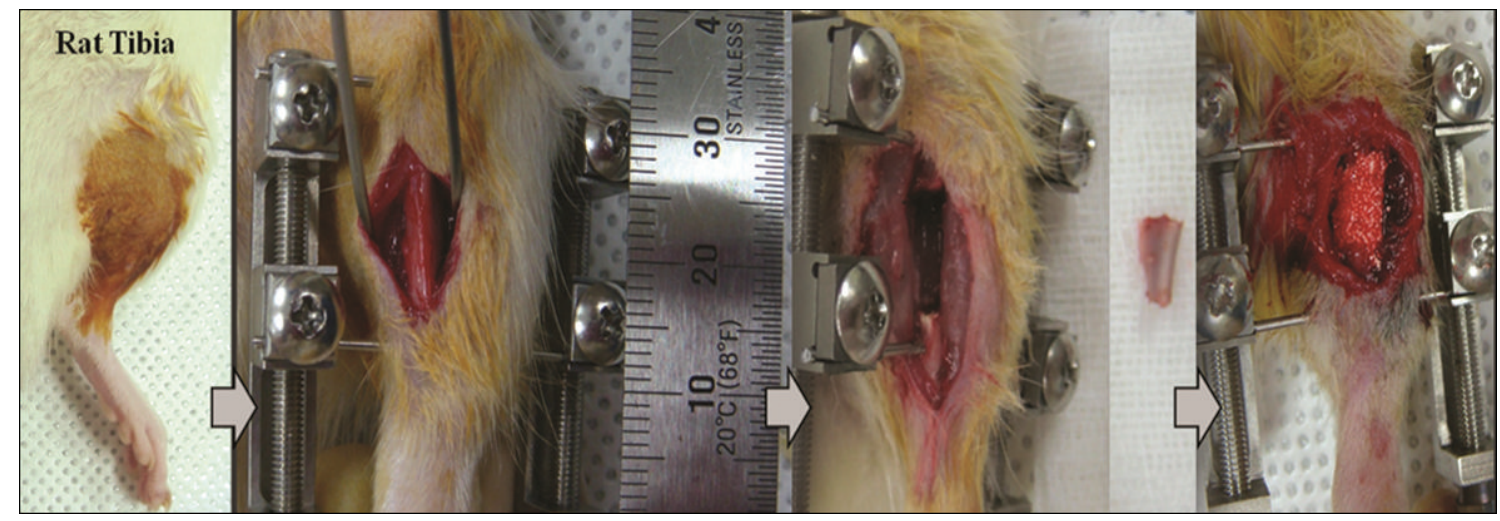

Figure 1: Serial photographs show the surgical procedure. After shaving, the periosteum and soft tissue were carefully retracted and two $0.9 \mathrm{~mm}$ K-wires (Zimmer, Warsaw, IN) were fixed to the tibia. K-wires were clamped bilaterally with the author's own-designed external fixator (U\&I, Gyeonggi-do, Korea). An $8 \mathrm{~mm}$ defect of the right tibia was created with a cutting burr, and $150 \mu \mathrm{L}$ of $\mathrm{CMC} / \mathrm{BioC}, \mathrm{CMC} / \mathrm{BioC} / \mathrm{BMP}-2$ $(0.1 \mathrm{mg})$, or CMC/BioC/BMP-2 $(0.5 \mathrm{mg})$ was injected into the defect, respectively.

were assessed with H\&E staining. Mineralized bone matrix and osteoid were evaluated by Masson's trichrome staining.

2.3. Statistical Analysis. Data are presented as mean \pm standard deviation. Statistical comparisons were carried out via one-way analysis of variance using Systat software (Chicago, IL, USA). Differences were considered statistically significant at ${ }^{*} P<0.05$ and ${ }^{* *} P<0.001$.

\section{Results}

3.1. In Vitro BMP-2 Release Study. On the first day, the released amounts of BMP-2 were $71.55 \pm 2.24 \mathrm{ng}$ with $\mathrm{CMC} / \mathrm{BioC} / \mathrm{BMP}-2(0.1 \mathrm{mg})$ and $83.75 \pm 1.12 \mathrm{ng}$ with CMC/Bio-C/BMP-2 (0.5 mg) (Figure 2). At 28 days, the released amounts of BMP-2 were $114.82 \pm 12.55 \mathrm{ng}$ with $\mathrm{CMC} / \mathrm{BioC} / \mathrm{BMP}-2(0.1 \mathrm{mg})$ and $151.76 \pm 9.57 \mathrm{ng}$ with CMC/Bio-C/BMP-2 (0.5 mg).

3.2. Plain Radiographs and Micro-CT Analysis. At 4 weeks after surgery, the plain radiographs revealed no mineralization at the defect area for the $\mathrm{CMC} / \mathrm{BioC}$ group but slight mineralization for the CMC/BioC/BMP-2 $(0.1 \mathrm{mg})$ and $\mathrm{CMC} / \mathrm{BioC} / \mathrm{BMP}-2(0.5 \mathrm{mg})$ groups (Figure 3$)$. At 8 weeks after surgery, both the CMC/BioC/BMP-2 $(0.1 \mathrm{mg})$ and CMC/BioC/BMP-2 (0.5 mg) groups showed much more mineralization than did the $\mathrm{CMC} / \mathrm{BioC}$ group. All three groups showed much greater mineralization at 8 weeks than at 4 weeks. The micro-CT images revealed that the defect areas for both CMC/BioC/BMP-2 (0.1 mg) and CMC/BioC/BMP-2 $(0.5 \mathrm{mg})$ showed slight mineralization while those for CMC/ BioC did not show mineralization at 4 weeks (Figure 4). In addition, more mineralization was visible in the $\mathrm{CMC} /$ BioC/BMP-2 $(0.5 \mathrm{mg})$ group than in the $\mathrm{CMC} / \mathrm{BioC}$ or $\mathrm{CMC} / \mathrm{BioC} / \mathrm{BMP}-2(0.1 \mathrm{mg})$ group at 8 weeks. Micro-CT revealed bone formation and bone remodeling. As shown in Figure 5(a), bone formation at the defect area was significantly greater for the $\mathrm{CMC} / \mathrm{BioC} / \mathrm{BMP}-2(0.5 \mathrm{mg})$ group

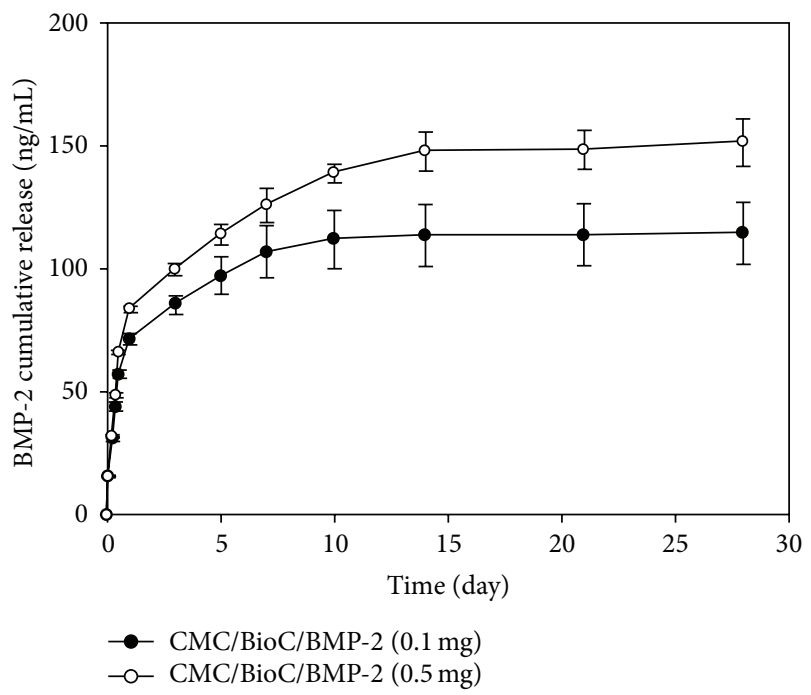

FIGURE 2: In vitro release profile of BMP-2 from CMC/BioC/BMP-2 hybrid materials. BMP-2 $(\bullet)$ from CMC/BioC/BMP-2 $(0.1 \mathrm{mg})$ and BMP-2 (O) from CMC/BioC/BMP-2 $(0.5 \mathrm{mg})$ hybrid materials. The error bars represent mean $\pm \mathrm{SD}(n=5)$. These experiments were repeated three times.

at 4 weeks than for the $\mathrm{CMC} / \mathrm{BioC}$ and $\mathrm{CMC} / \mathrm{BioC} / \mathrm{BMP}-2$ $(0.1 \mathrm{mg})$ groups $\left({ }^{* *} \mathrm{P}<0.001\right)$. A significant difference in bone formation was observed between the $\mathrm{CMC} / \mathrm{BioC} / \mathrm{BMP}$ 2 group $(0.5 \mathrm{mg})$ and $\mathrm{CMC} / \mathrm{BioC}$ group $\left({ }^{* *} P<0.001\right)$ and between the CMC/BioC/BMP-2 group $(0.5 \mathrm{mg})$ and the CMC/BioC/BMP-2 $(0.1 \mathrm{mg})$ group $\left({ }^{*} P<0.05\right)$ at 8 weeks. At 4 weeks, there was a statistically significant difference in bone remodeling of the defect area between the $\mathrm{CMC} / \mathrm{BioC} / \mathrm{BMP}-2$ group $(0.5 \mathrm{mg})$ and the $\mathrm{CMC} / \mathrm{BioC}$ group $\left({ }^{*} P<0.05\right)$, as well as between the CMC/BioC/BMP-2 group $(0.5 \mathrm{mg})$ and the CMC/BioC/BMP-2 $(0.1 \mathrm{mg})$ group $\left({ }^{*} P<0.05\right)$ (Figure 5(b)). In addition, a significantly greater amount of bone remodeling occurred in the CMC/BioC/BMP-2 $(0.5 \mathrm{mg})$ group than either the $\mathrm{CMC} / \mathrm{BioC}$ group $\left({ }^{* *} P<0.001\right)$ or the CMC/BioC/BMP-2 $(0.1 \mathrm{mg})$ group at 8 weeks $\left({ }^{*} P<0.05\right)$. 


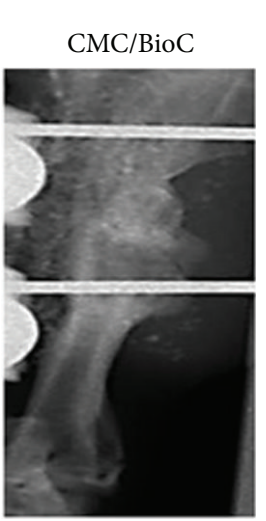

(a)

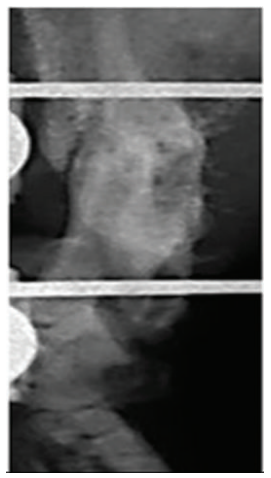

(d)

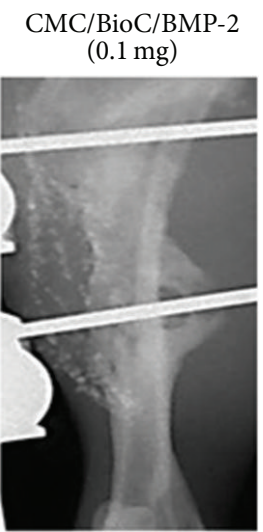

(b)

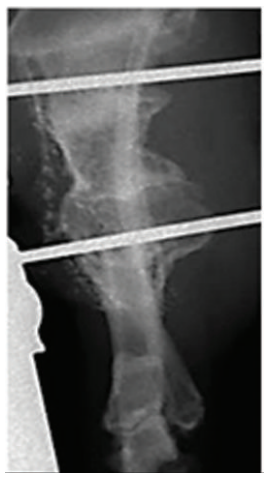

(e)
CMC/BioC/BMP-2 (0.5 mg)

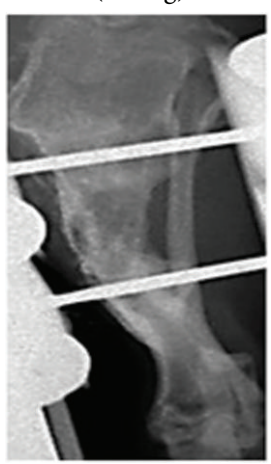

(c)

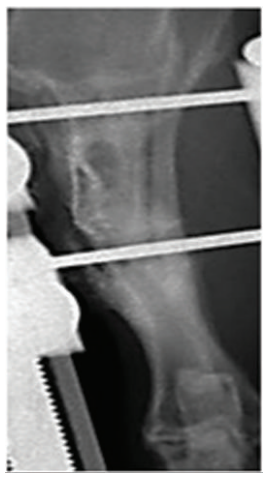

(f)

FIGURE 3: Plain radiographs of (a) CMC/BioC, (b) CMC/BioC/BMP-2 (0.1 mg), and (c) CMC/BioC/BMP-2 (0.5 mg) at 4 weeks after implantation onto the tibial defects. (d) CMC/BioC, (e) CMC/BioC/BMP-2 (0.1 mg), and (f) CMC/BioC/BMP-2 (0.5 mg) at 8 weeks after implantation onto the tibial defects. There was no mineralization at the defect site after the implantation of $\mathrm{CMC} / \mathrm{BioC}$, whereas both CMC/BioC/BMP-2 $(0.1 \mathrm{mg})$ and CMC/BioC/BMP-2 $(0.5 \mathrm{mg})$ experienced slight mineralization at 4 weeks. At 8 weeks after surgery, both the $\mathrm{CMC} / \mathrm{BioC} / \mathrm{BMP}-2(0.1 \mathrm{mg})$ and $\mathrm{CMC} / \mathrm{BioC} / \mathrm{BMP}-2(0.5 \mathrm{mg})$ groups experienced much greater mineralization than did the $\mathrm{CMC} / \mathrm{BioC}$ group.

3.3. Histological Study. The H\&E staining analysis revealed that the tissue of the defect area showed negligible mineralized bone tissue for the $\mathrm{CMC} / \mathrm{BioC}$ group and partially mineralized bone tissue for the CMC/BioC/BMP-2 (0.1 mg) group. However, the tissue of the defect area showed a much greater amount of mineralized bone tissue for the $\mathrm{CMC} / \mathrm{BioC} / \mathrm{BMP}-2(0.5 \mathrm{mg})$ group than for the $\mathrm{CMC} / \mathrm{BioC}$ or $\mathrm{CMC} / \mathrm{BioC} / \mathrm{BMP}-2(0.1 \mathrm{mg})$ group at 4 weeks (Figures $6(\mathrm{a})-6(\mathrm{c}))$. In all three groups, a much greater amount of mineralized bone tissue was observed around the defect at 8 weeks after surgery than at 4 weeks after surgery. Moreover, mineralized bone tissue was observed at the whole circumference of the defect site in the CMC/BioC/BMP-2 (0.5 mg) group but not in the $\mathrm{CMC} / \mathrm{BioC}$ or $\mathrm{CMC} / \mathrm{BioC} / \mathrm{BMP}-2$ $(0.1 \mathrm{mg})$ group at 8 weeks (Figures $6(\mathrm{~d})-6(\mathrm{f}))$. The Masson's trichrome staining analysis revealed that general woven bone tissue covered the defects in the $\mathrm{CMC} / \mathrm{BioC}$ group, whereas at least some mineralized bone tissue was present in the CMC/BioC/BMP-2 (0.1 mg) and CMC/BioC/BMP-2 (0.5 mg) groups at 4 weeks (Figures $7(\mathrm{a})-7(\mathrm{c})$ ). At 8 weeks, much of the mineralized bone tissue on the defect site was visible in the CMC/BioC/BMP-2 (0.5 mg) group, but this amount was much lower in the CMC/BioC or CMC/BioC/BMP-2 (0.1 mg) group (Figures $7(\mathrm{~d})-7(\mathrm{f})$ ).

\section{Discussion}

Osteoconductive materials, such as CaP-based materials, allow a framework for vascular invasion and cellular infiltration but do not induce mesenchymal cells to differentiate into mature bone cells. Osteoinductive materials, including growth factors such as BMPs, basic fibroblast growth factor (bFGF), platelet-derived growth factor (PDGF), and vascular endothelial growth factor (VEGF), induce new bone formation but they do not provide a framework for vascular invasion and cellular infiltration. Thus, osteoconductive materials combined with osteoinductive materials may be ideal for bone regeneration.

The objective of this study was to assess whether a CMCbased hydrogel containing BioC and BMP-2 induced a greater amount of new bone formation than did a CMC-based hydrogel containing BioC without BMP-2. The results from the plain radiographs and micro-CT demonstrated that bone formation was significantly greater in the CMC/BioC/BMP-2 


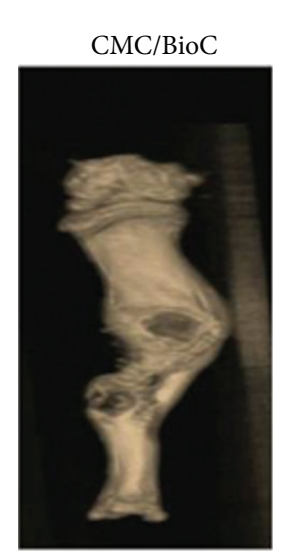

(a)

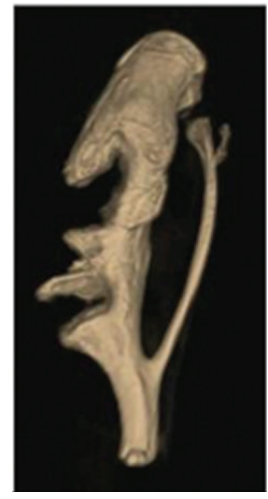

[t!]

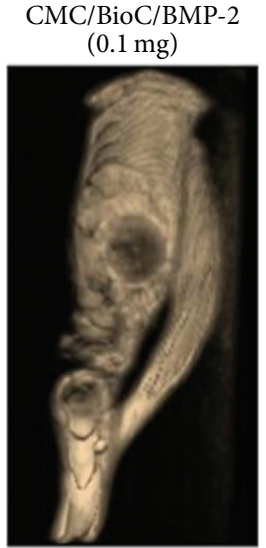

(b)

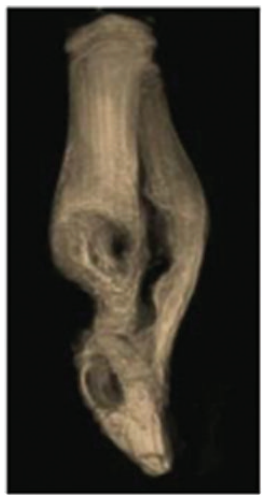

(e)
CMC/BioC/BMP-2 $(0.5 \mathrm{mg})$

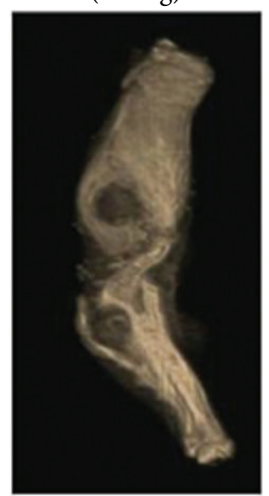

(c)

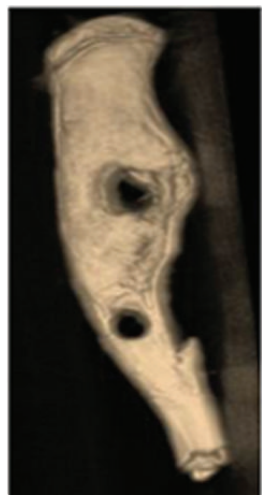

(f)

Figure 4: Micro-CT images of (a) CMC/BioC, (b) CMC/BioC/BMP-2 (0.1 mg), and (c) CMC/BioC/BMP-2 (0.5 mg) at 4 weeks after implantation onto the tibial defects. (d) CMC/BioC, (e) CMC/BioC/BMP-2 (0.1 mg), and (f) CMC/BioC/BMP-2 (0.5 mg) at $8 \mathrm{weeks}$ after implantation onto the tibial defects. The micro-CT images revealed slight mineralization at the defect site for both the CMC/BioC/BMP-2 $(0.1 \mathrm{mg})$ and $\mathrm{CMC} / \mathrm{BioC} / \mathrm{BMP}-2(0.5 \mathrm{mg})$ groups but no mineralization for the CMC/BioC group at 4 weeks. At 8 weeks, mineralization was much more extensive in the CMC/BioC/BMP-2 (0.5 mg) group than in the CMC/BioC or CMC/BioC/BMP-2 (0.1 mg) group at 8 weeks.

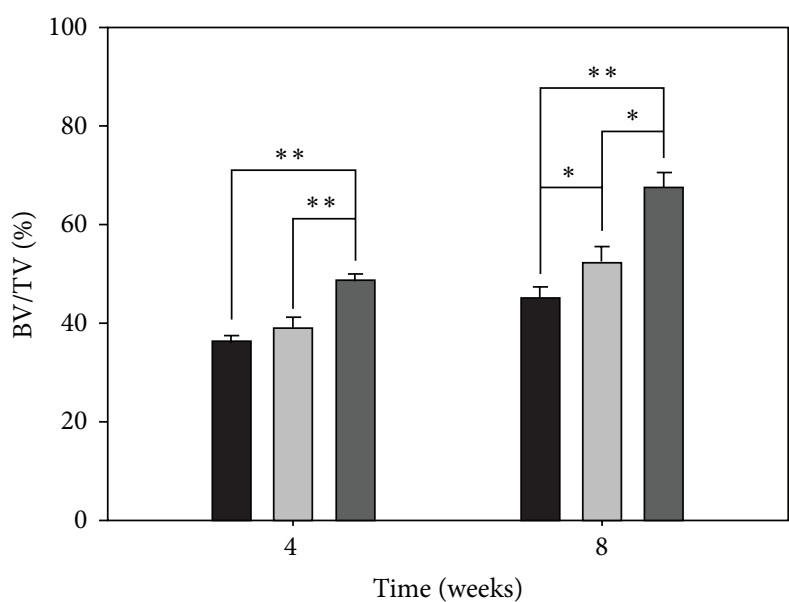

$\mathrm{CMC} / \mathrm{BioC}$

CMC/BioC/BMP-2 (0.1 mg) CMC/BioC/BMP-2 (0.5 mg)

(a)

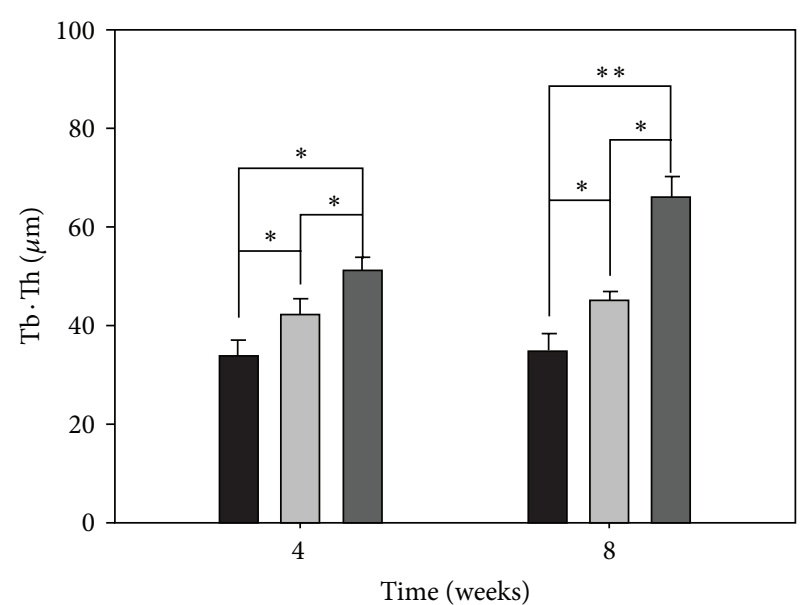

$\mathrm{CMC} / \mathrm{BioC}$

CMC/BioC/BMP-2 (0.1 mg) CMC/BioC/BMP-2 (0.5 mg)

(b)

Figure 5: (a) Bone formation and (b) bone remodeling for the CMC/BioC, CMC/BioC/BMP-2 (0.1 mg), and CMC/BioC/BMP-2 (0.5 mg) groups at 4 and 8 weeks. The values represent mean \pm standard deviation $(n=12)\left({ }^{*} P<0.05\right.$ and $\left.{ }^{* *} P<0.001\right)$. 


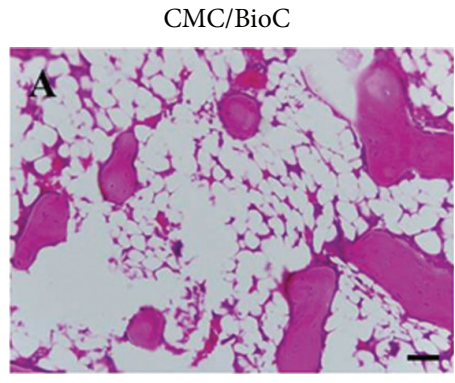

(a)

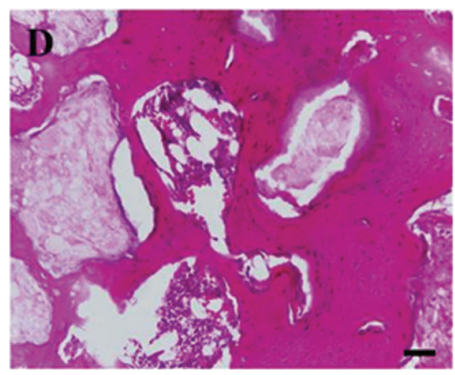

(d)
CMC/BioC/BMP-2 (0.1 mg)

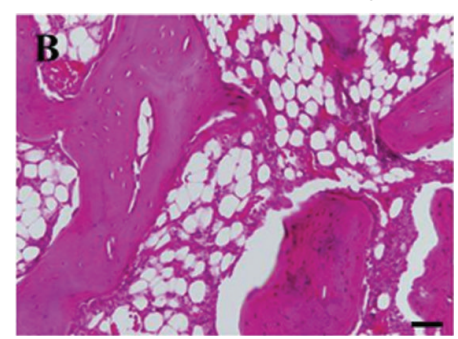

(b)

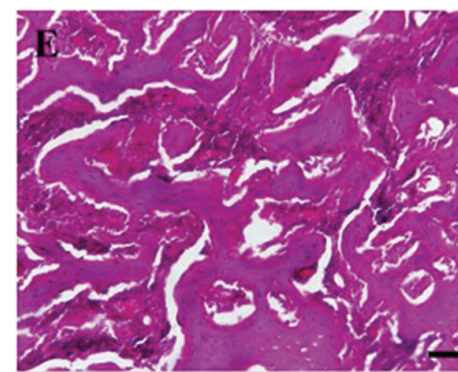

(e)
CMC/BioC/BMP-2 (0.5 mg)

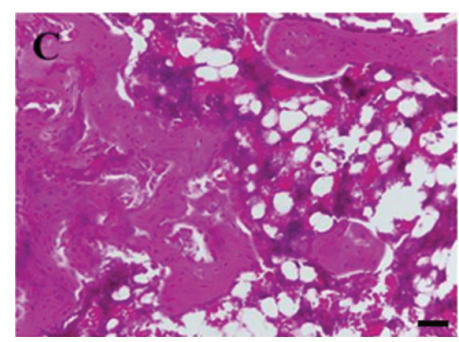

(c)

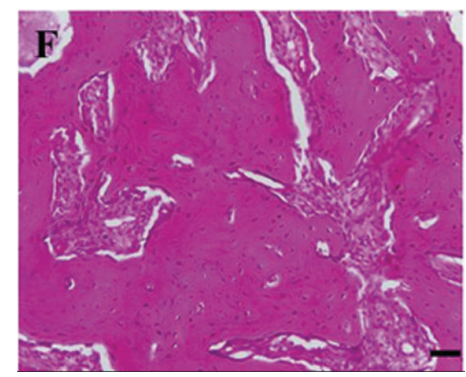

(f)

Figure 6: H\&E staining of the rat tibial defect after implantation of (a) CMC/BioC, (b) CMC/BioC/BMP-2 (0.1 mg), and (c) CMC/BioC/BMP$2(0.5 \mathrm{mg})$ at 4 weeks. (d) $\mathrm{CMC} / \mathrm{BioC}$, (e) CMC/BioC/BMP-2 (0.1 mg), and (f) CMC/BioC/BMP-2 (0.5 mg) at 8 weeks. The tissue of the defect area showed a much greater amount of mineralized bone tissue for the CMC/BioC/BMP-2 (0.5 mg) group than for the CMC/BioC or CMC/BioC/BMP-2 $(0.1 \mathrm{mg})$ group at 4 weeks. Mineralized bone tissue was observed at the whole circumference of the defect site in the $\mathrm{CMC} / \mathrm{BioC} / \mathrm{BMP}-2(0.5 \mathrm{mg})$ group but not in the CMC/BioC or CMC/BioC/BMP-2 $(0.1 \mathrm{mg})$ group at 8 weeks $(\mathrm{scale}$ bar $=50 \mu \mathrm{m})$.

$\mathrm{CMC} / \mathrm{BioC}$

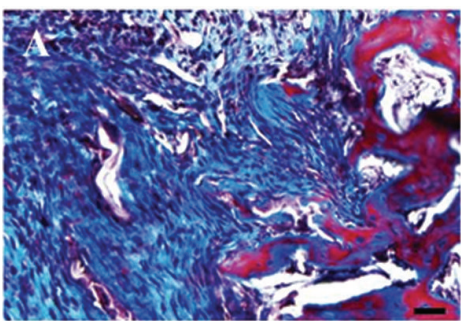

(a)

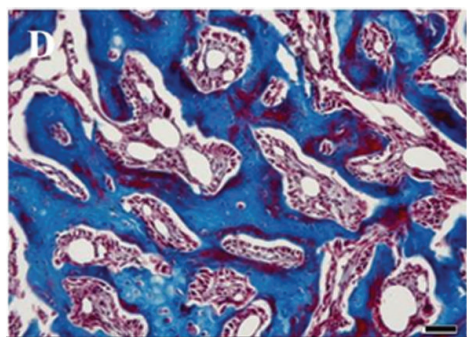

(d)
$\mathrm{CMC} / \mathrm{BioC} / \mathrm{BMP}-2(0.1 \mathrm{mg})$

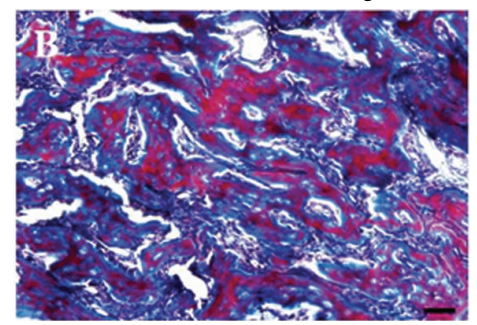

(b)

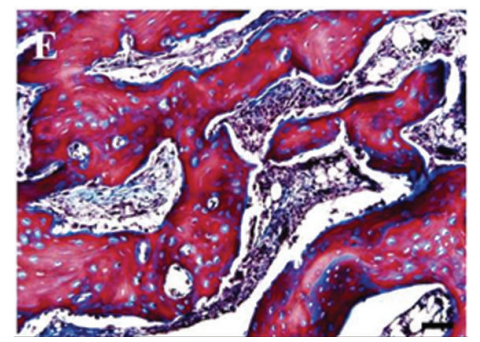

(e)
$\mathrm{CMC} / \mathrm{BioC} / \mathrm{BMP}-2(0.5 \mathrm{mg})$

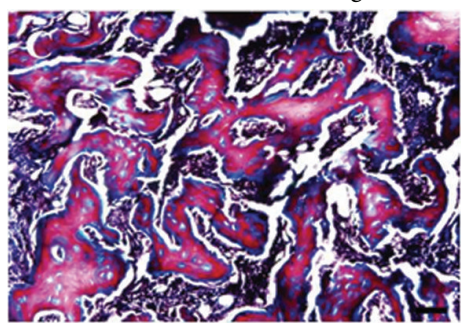

(c)

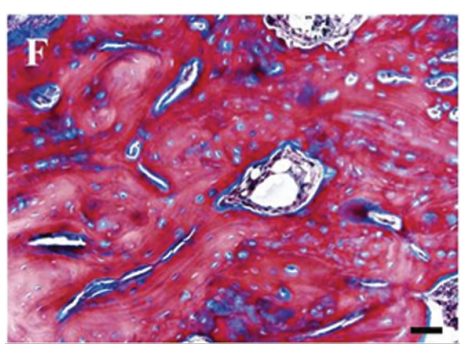

(f)

FIGURE 7: Masson's trichrome staining of the rat tibial defect after implantation of (a) CMC/BioC, (b) CMC/BioC/BMP-2 (0.1 mg), and (c) CMC/BioC/BMP-2 (0.5 mg) at 4 weeks. (d) CMC/BioC, (e) CMC/BioC/BMP-2 (0.1 mg), and (f) CMC/BioC/BMP-2 (0.5 mg) at 8 weeks. General woven bone tissue (blue color) covered the defects in the $\mathrm{CMC} / \mathrm{BioC}$ group, whereas at least some mineralized bone tissue (red color) was present in the CMC/BioC/BMP-2 $(0.1 \mathrm{mg})$ and CMC/BioC/BMP-2 $(0.5 \mathrm{mg})$ groups at 4 weeks. At 8 weeks, much of the mineralized bone tissue on the defect site was visible in the CMC/BioC/BMP-2 $(0.5 \mathrm{mg})$ group, but this amount was much lower in the $\mathrm{CMC} / \mathrm{BioC}$ or CMC/BioC/BMP-2 (0.1 mg) group (scale bar $=50 \mu \mathrm{m})$. 
(0.5 mg) group than in the $\mathrm{CMC} / \mathrm{BioC}$ or $\mathrm{CMC} / \mathrm{BioC} / \mathrm{BMP}-$ $2(0.1 \mathrm{mg})$ group at 4 and 8 weeks. Thus, these findings demonstrate that CMC/BioC/BMP-2 $(0.5 \mathrm{mg})$ induces rapid bone formation at an earlier stage than does $\mathrm{CMC} / \mathrm{BioC}$ or CMC/BioC/BMP-2 (0.1 mg). As expected from the plain radiographs and micro-CT data, the histological study data revealed that the $\mathrm{CMC} / \mathrm{BioC} / \mathrm{BMP}-2(0.5 \mathrm{mg})$ group had more mineralized bone tissue at the defect site than did the $\mathrm{CMC} / \mathrm{BioC}$ and $\mathrm{CMC} / \mathrm{BioC} / \mathrm{BMP}-2(0.1 \mathrm{mg})$ groups at 4 weeks. In all groups, a greater amount of mineralized bone tissue was observed at the defect site at 8 weeks than at 4 weeks. The entire defect area was covered with mineralized bone tissue at 8 weeks in the CMC/BioC/BMP-2 $(0.5 \mathrm{mg})$ group but not in the CMC/BioC or CMC/BioC/BMP-2 (0.1 mg) group.

These results are consistent with those of previous studies. Lin et al. [28] reported that BMP-2-immobilized heparinbound demineralized bone matrix (HC-DBM) showed higher alkaline phosphatase (ALP) activity (2 weeks), more calcium deposition ( 4 and 8 weeks), and more bone formation than that of controls after subcutaneous implantation in rats. Zhao et al. [29] demonstrated that a BMP-2absorbed monoclonal antibodies conjugated DBM (MAbsDBM) group experienced greater osteogenic differentiation in an in vitro study and greater ectopic bone formation in an in vivo study than the control group. In a previous study, we found that woven bone covered the whole circumference more often in a BMP-2-coated tricalcium phosphate/hydroxyapatite group than in a tricalcium phosphate/hydroxyapatite group in a rat model of femoral distraction osteogenesis [30, 31]. Kim et al. [32] and Park et al. [33] reported that BMP-2-coated biphasic calcium phosphate (BCP) granules or blocks supported significantly greater bone formation than BCP granules or blocks in a rat model of calvarial defects. Moreover, Choi et al. [34] showed that implantation of BMP-2/BCP granules onto $6 \mathrm{~mm}$ diameter defects of the maxillary sinus of rabbits led to enhanced bone formation compared with the control group. Finally, a recent report demonstrated that new bone formation at a bone defect in the middle ear after mastoid surgery was greater in the presence of BMP-2/BCP scaffolds [35].

Our results suggest that $\mathrm{CMC} / \mathrm{BioC} / \mathrm{BMP}-2$ hybrid materials induce greater bone formation at an earlier stage through release of BMP-2 than CMC/BioC hybrid materials. However, our in vivo study has some limitations. For example, longterm evaluation greater than 8 weeks is needed to compare the quality and architecture of new bone formation between control and implanted groups. More comprehensive analyses that include the histology and angiogenesis of new bone and biomechanical testing are needed. Finally, the effects of longterm release of BMP- 2 require further investigation.

In conclusion, CMC/BioC/BMP-2 (0.5 mg) hybrid materials implanted in a rat tibial defect model led to greater bone formation than did $\mathrm{CMC} / \mathrm{BioC}$ and $\mathrm{CMC} / \mathrm{BioC} / \mathrm{BMP}-$ 2 (0.1 mg) hybrid materials. Thus, CMC/BioC/BMP-2 hybrid materials may be useful in an injectable substrate for the clinical application of the regeneration of bone defects in the orthopedic field.

\section{Conflict of Interests}

The authors declare that there is no conflict of interests regarding the publication of this paper.

\section{Authors' Contribution}

Sang-Heon Song and Young-Pil Yun contributed equally to this paper.

\section{Acknowledgment}

This study was supported by a Grant from the Korea Healthcare Technology R\&D Project, Ministry of Health and Welfare, Republic of Korea (HI11C0388).

\section{References}

[1] B. M. Chesnutt, Y. Yuan, K. Buddington, W. O. Haggard, and J. D. Bumgardner, "Composite chitosan/nano-hydroxyapatite scaffolds induce osteocalcin production by osteoblasts in vitro and support bone formation in vivo," Tissue Engineering A, vol. 15, no. 9, pp. 2571-2579, 2009.

[2] P. V. Giannoudis, H. Dinopoulos, and E. Tsiridis, "Bone substitutes: an update," Injury, vol. 36, supplement 3, pp. S20-S27, 2005.

[3] T. Kurien, R. G. Pearson, and B. E. Scammell, "Bone graft substitutes currently available in orthopaedic practice: the evidence for their use," The Bone and Joint Journal B, vol. 95, no. 5, pp. 583-597, 2013.

[4] R. Z. LeGeros, "Calcium phosphate-based osteoinductive materials," Chemical Reviews, vol. 108, no. 11, pp. 4742-4753, 2008.

[5] E. A. Cowles, M. E. DeRome, G. Pastizzo, L. L. Brailey, and G. A. Gronowicz, "Mineralization and the expression of matrix proteins during in vivo bone development," Calcified Tissue International, vol. 62, no. 1, pp. 74-82, 1998.

[6] S. Stevenson, N. Cunningham, J. Toth, D. Davy, and A. H. Reddi, "The effect of osteogenin (a bone morphogenetic protein) on the formation of bone in orthotopic segmental defects in rats," The Journal of Bone and Joint Surgery A, vol. 76, no. 11, pp. 1676$1687,1994$.

[7] J. D. Termine, H. K. Kleinman, and W. Whitson, "Osteonectin, a bone-specific protein linking mineral to collagen," Cell, vol. 26, no. 1, pp. 99-105, 1981.

[8] M. R. Urist, "Bone morphogenetic protein: the molecularization of skeletal system development," Journal of Bone and Mineral Research, vol. 12, no. 3, pp. 343-346, 1997.

[9] J. M. Wozney, "The bone morphogenetic protein family and osteogenesis," Molecular Reproduction and Development, vol. 32, no. 2, pp. 160-167, 1992.

[10] O. A. Arosarena and D. Puleo, "In vitro effects of combined and sequential bone morphogenetic protein administration," Archives of Facial Plastic Surgery, vol. 9, no. 4, pp. 242-247, 2007.

[11] S. D. Cook, M. W. Wolfe, S. L. Salkeld, and D. C. Rueger, "Effect of recombinant human osteogenic protein-1 on healing of segmental defects in non-human primates," The Journal of Bone and Joint Surgery A, vol. 77, no. 5, pp. 734-750, 1995.

[12] S. E. Kim, O. Jeon, J. B. Lee et al., "Enhancement of ectopic bone formation by bone morphogenetic protein-2 delivery using heparin-conjugated PLGA nanoparticles with transplantation 
of bone marrow-derived mesenchymal stem cells," Journal of Biomedical Science, vol. 15, no. 6, pp. 771-777, 2008.

[13] E. A. Wang, V. Rosen, J. S. D’Alessandro et al., "Recombinant human bone morphogenetic protein induces bone formation," Proceedings of the National Academy of Sciences of the United States of America, vol. 87, no. 6, pp. 2220-2224, 1990.

[14] D. L. Diefenderfer, A. M. Osyczka, J. P. Garino, and P. S. Leboy, "Regulation of BMP-induced transcription in cultured human bone marrow stromal cells," The Journal of Bone and Joint Surgery A, vol. 85, supplement 3, pp. 19-28, 2003.

[15] F. Lecanda, L. V. Avioli, and S. L. Cheng, "Regulation of bone matrix protein expression and induction of differentiation of human osteoblasts and human bone marrow stromal cells by bone morphogenetic protein-2," Journal of Cellular Biochemistry, vol. 67, no. 3, pp. 386-396, 1997.

[16] P. Chua, K. Neoh, E. Kang, and W. Wang, "Surface functionalization of titanium with hyaluronic acid/chitosan polyelectrolyte multilayers and RGD for promoting osteoblast functions and inhibiting bacterial adhesion," Biomaterials, vol. 29, no. 10, pp. 1412-1421, 2008.

[17] J. Kim, I. S. Kim, T. H. Cho et al., "Bone regeneration using hyaluronic acid-based hydrogel with bone morphogenic protein-2 and human mesenchymal stem cells," Biomaterials, vol. 28, no. 10, pp. 1830-1837, 2007.

[18] G. N. King, N. King, A. T. Cruchley, J. M. Wozney, and F. J. Hughes, "Recombinant human Bone Morphogenetic Protein2 promotes wound healing in rat periodontal fenestration defects," Journal of Dental Research, vol. 76, no. 8, pp. 1460-1470, 1997.

[19] T. Lee, J. Ho, K. Hung, W. Chen, Y. Chung, and Y. Yang, "Bone morphogenetic protein gene therapy using a fibrin scaffold for a rabbit spinal-fusion experiment," Neurosurgery, vol. 58, no. 2, pp. 373-380, 2006.

[20] Z. Shi, K. G. Neoh, E. Kang, C. Poh, and W. Wang, "Titanium with surface-grafted dextran and immobilized bone morphogenetic protein-2 for inhibition of bacterial adhesion and enhancement of osteoblast functions," Tissue Engineering A, vol. 15 , no. 2, pp. 417-426, 2009.

[21] G. Zellin and A. Linde, "Importance of delivery systems for growth-stimulatory factors in combination with osteopromotive membranes. An experimental study using rhBMP-2 in rat mandibular defects," Journal of Biomedical Materials Research, vol. 35, no. 2, pp. 181-190, 1997.

[22] N. A. Ramli and T. W. Wong, "Sodium carboxymethylcellulose scaffolds and their physicochemical effects on partial thickness wound healing," International Journal of Pharmaceutics, vol. 403, no. 1-2, pp. 73-82, 2011.

[23] A. T. Reza and S. B. Nicoll, "Characterization of novel photocrosslinked carboxymethylcellulose hydrogels for encapsulation of nucleus pulposus cells," Acta Biomaterialia, vol. 6, no. 1, pp. 179-186, 2010.

[24] P. G. Bowler, S. A. Jones, B. J. Davies, and E. Coyle, "Infection control properties of some wound dressings," Journal of Wound Care, vol. 8, no. 10, pp. 499-502, 1999.

[25] C. K. Ryan and H. C. Sax, "Evaluation of a carboxymethylcellulose sponge for prevention of postoperative adhesions," The American Journal of Surgery, vol. 169, no. 1, pp. 154-160, 1995.

[26] G. Leone, M. Fini, P. Torricelli, R. Giardino, and R. Barbucci, "An amidated carboxymethylcellulose hydrogel for cartilage regeneration," Journal of Materials Science: Materials in Medicine, vol. 19, no. 8, pp. 2873-2880, 2008.
[27] D. Pasqui, P. Torricelli, M. de Cagna, M. Fini, and R. Barbucci, "Carboxymethyl cellulose-hydroxyapatite hybrid hydrogel as a composite material for bone tissue engineering applications," Journal of Biomedical Materials Research A, 2013.

[28] H. Lin, Y. Zhao, W. Sun et al., "The effect of crosslinking heparin to demineralized bone matrix on mechanical strength and specific binding to human bone morphogenetic protein-2," Biomaterials, vol. 29, no. 9, pp. 1189-1197, 2008.

[29] Y. Zhao, J. Zhang, X. Wang et al., "The osteogenic effect of bone morphogenetic protein- 2 on the collagen scaffold conjugated with antibodies," Journal of Controlled Release, vol. 141, no. 1, pp. 30-37, 2010.

[30] H. R. Song, S. E. Kim, H. G. Kim, Y. P. Yun, J. H. Bae, and H. J. Kim, "The effects of rhBMP-2 injection at distraction osteogenesis of Rats' Tibia," Tissue Engineering and Regenerative Medicine, vol. 8, no. 2, pp. 158-163, 2011.

[31] J. Yang, H. Kim, S. Kim et al., "The effect of bone morphogenic protein-2-coated tri-calcium phosphate/hydroxyapatite on new bone formation in a rat model of femoral distraction osteogenesis," Cytotherapy, vol. 14, no. 3, pp. 315-326, 2012.

[32] J. Kim, K. Choi, J. Yun et al., "Bone formation of block and particulated biphasic calcium phosphate lyophilized with Escherichia coli-derived recombinant human bone morphogenetic protein 2 in rat calvarial defects," Oral Surgery, Oral Medicine, Oral Pathology, Oral Radiology and Endodontology, vol. 112, no. 3, pp. 298-306, 2011.

[33] J.-C. Park, S.-S. So, I.-H. Jung et al., "Induction of bone formation by Escherichia coli-expressed recombinant human bone morphogenetic protein-2 using block-type macroporous biphasic calcium phosphate in orthotopic and ectopic rat models," Journal of Periodontal Research, vol. 46, no. 6, pp. 682690, 2011.

[34] H. Choi, N. J. Park, O. Jamiyandorj et al., "Improvement of osteogenic potential of biphasic calcium phosphate bone substitute coated with two concentrations of expressed recombinant human bone morphogenetic protein 2," Journal of Periodontal and Implant Science, vol. 42, no. 4, pp. 119-126, 2012.

[35] E. Kuhnert, N. Ehlert, P. Behrens et al., "Different cell populations are inducible by BMP-2 covalently covered Bioverit(R) II implants in rabbit subcutis and middle ear," Biomedizinische Technik Biomedical Engineering, 2013. 


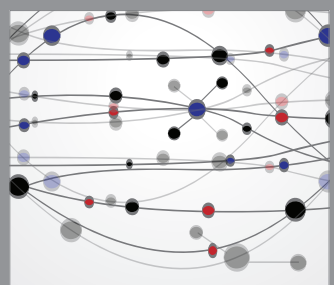

The Scientific World Journal
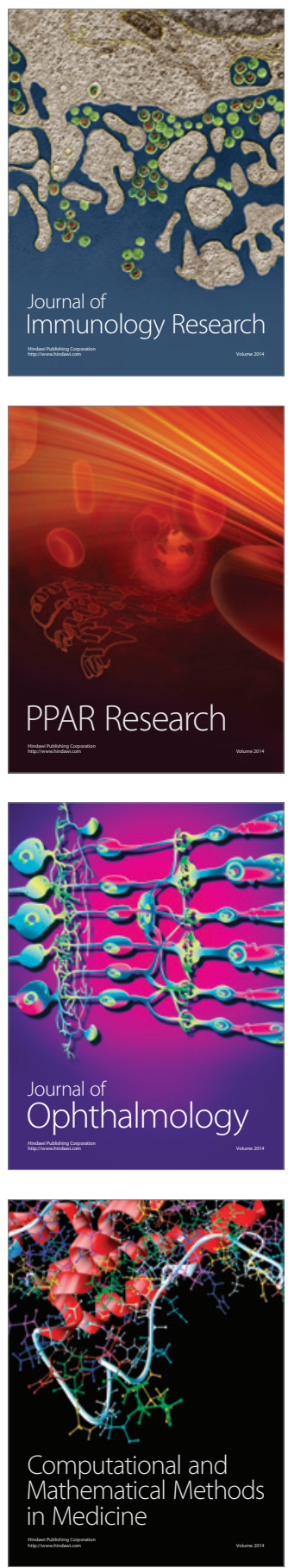

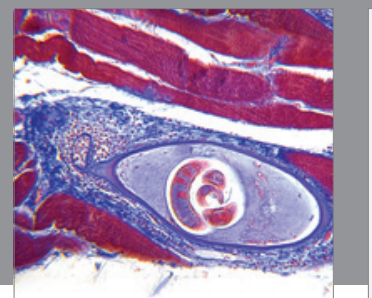

Gastroenterology

Research and Practice
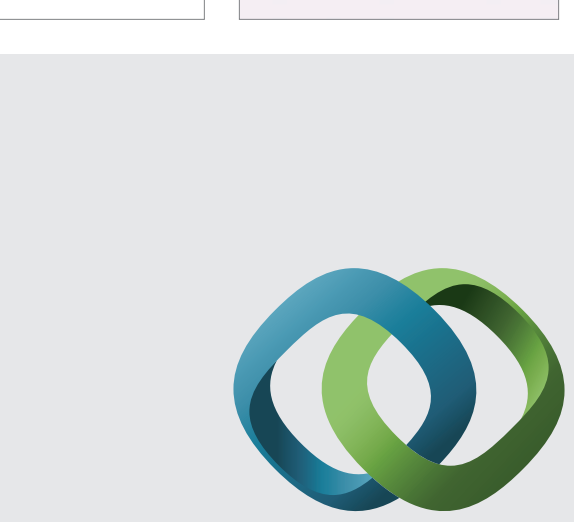

\section{Hindawi}

Submit your manuscripts at

http://www.hindawi.com
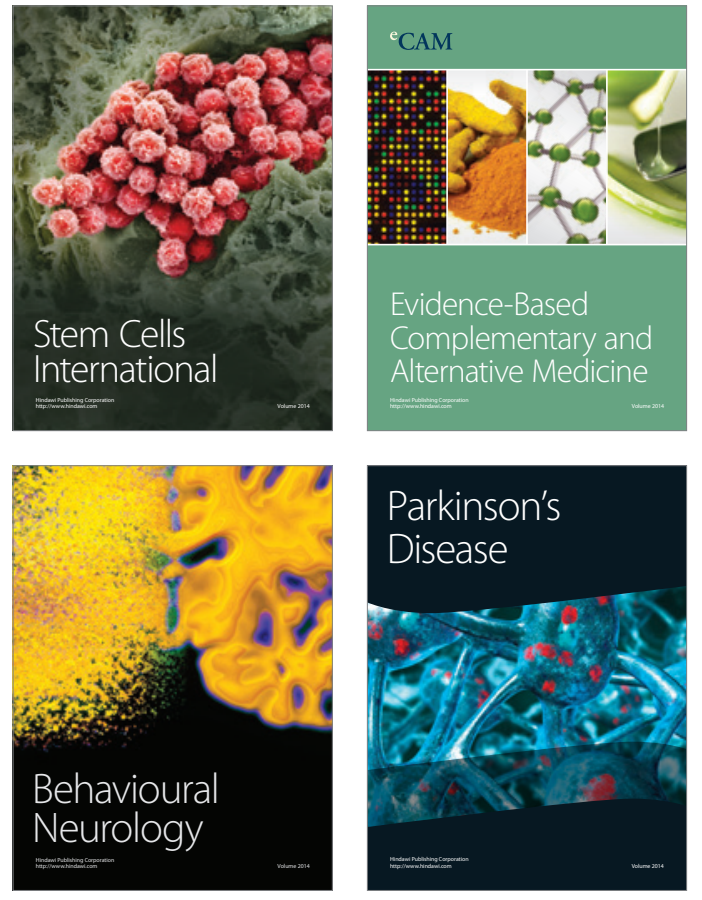
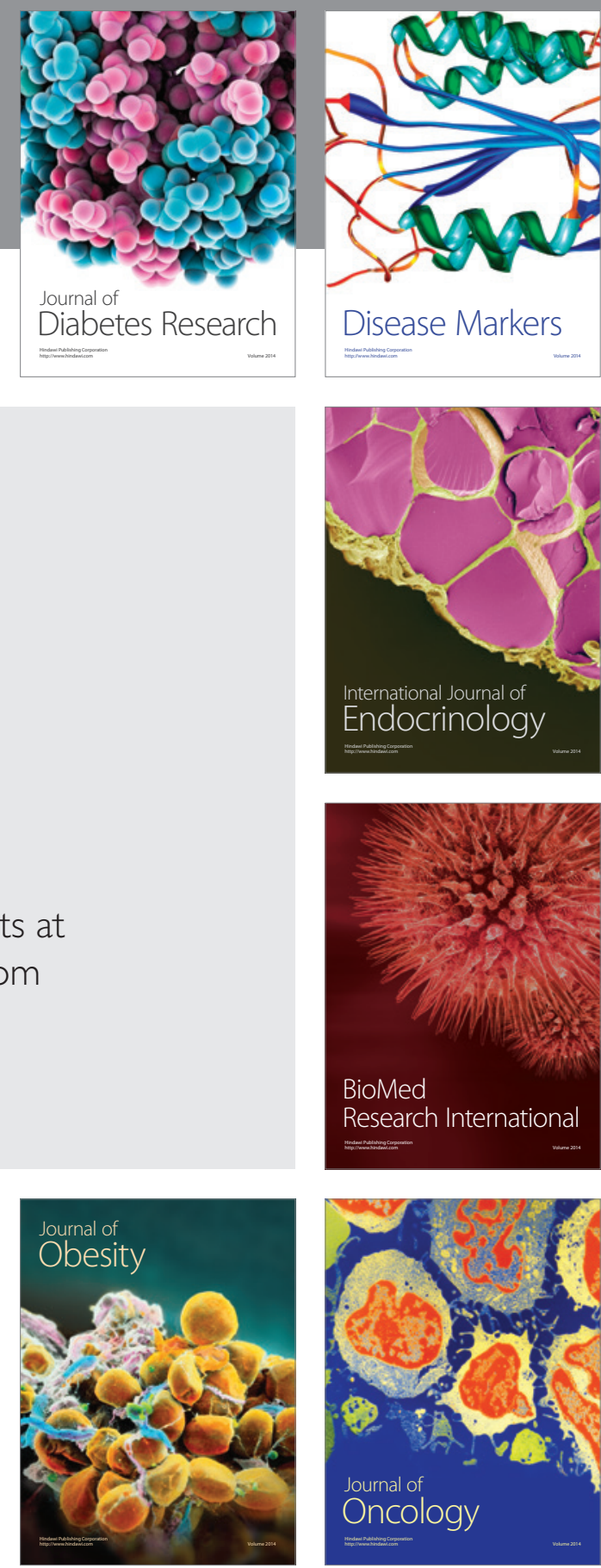

Disease Markers
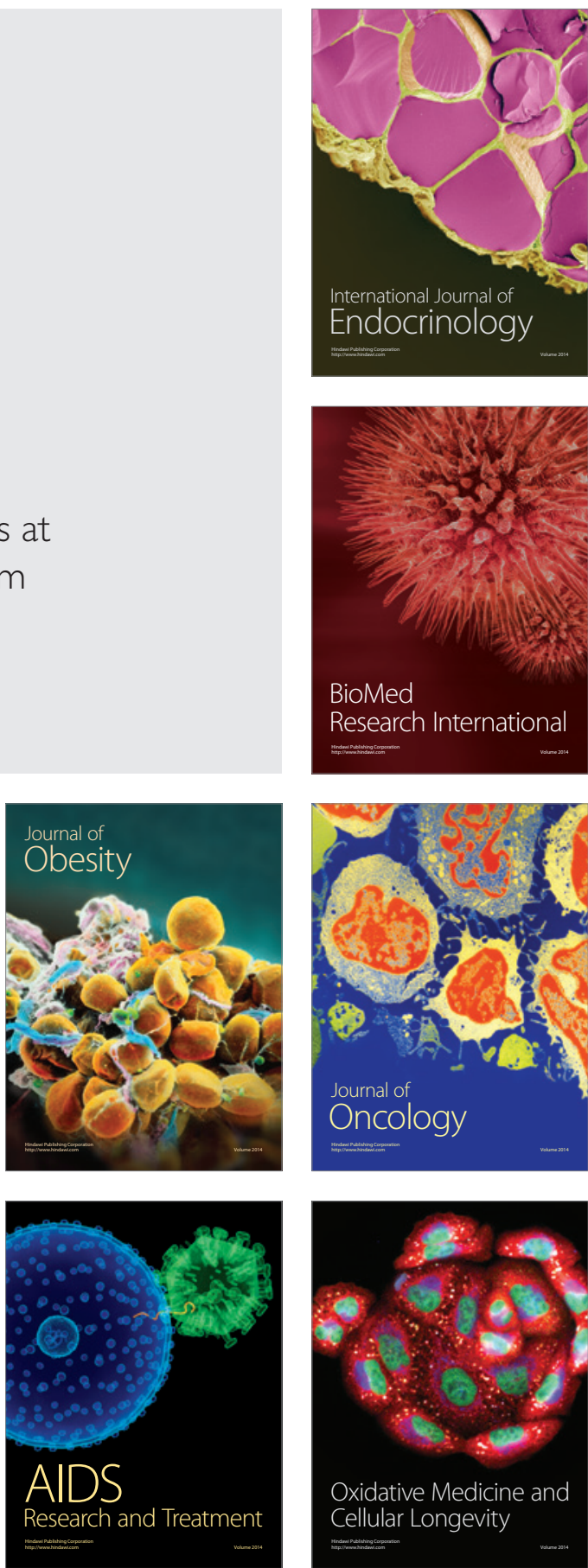